\title{
The Pass-on Effect of
}

\section{Tetracycline-Induced Honey Bee (Apis mellifera) Gut Community Dysbiosis}

\author{
Shuo Jia, Yuqi Wu, Gongwen Chen, Shuai Wang, Fuliang Hu and Huoqing Zheng* \\ College of Animal Sciences, Zhejiang University, Hangzhou, China
}

OPEN ACCESS

Edited by:

Simon Luke Elliot,

Universidade Federal de Viçosa, Brazil

Reviewed by:

Juris A. Grasis,

University of California, Merced,

United States

Beatriz Sabater-Munoz,

Institute of Molecular and Cellular

Biology of Plants, Polytechnic

University of Valencia, Spain

*Correspondence:

Huoqing Zheng

hqzheng@zju.edu.cn

Specialty section:

This article was submitted to

Microbial Symbioses,

a section of the journa

Frontiers in Microbiology

Received: 23 September 2021

Accepted: 28 December 2021

Published: 18 January 2022

Citation:

Jia S, Wu Y, Chen G, Wang S,

Hu F and Zheng H (2022) The

Pass-on Effect

of Tetracycline-Induced Honey Bee

(Apis mellifera) Gut Community

Dysbiosis.

Front. Microbiol. 12:781746.

doi: 10.3389/fmicb.2021.781746
Gut microbial community plays an important role in the regulation of insect health. Antibiotic treatment is powerful to fight bacterial infections, while it also causes collateral damage to gut microbiome, which may have long-lasting consequences for host health. However, current studies on honey bees mainly focus on the impact of direct exposure to antibiotics on individual bees, and little is known about the impact of social transmission of antibiotic-induced gut community disorder in honey bee colonies. In order to provide insight into the potential pass-on effect of antibiotic-induced dysbiosis, we colonized newly emerged germ-free workers with either normal or tetracyclinetreated gut community and analyzed the gut bacteria composition. We also treated workers with low dosage of tetracycline to evaluate its impact on honey bee gut microbiota. Our results showed that the tetracycline-treated gut community caused disruption of gut community in their receivers, while the direct exposure to the low dosage of tetracycline had no significant effect. In addition, no significant difference was observed on the mortality rate of $A$. mellifera workers with different treatments. These results suggest that though the residue of antibiotic treatment may not have direct effect on honey bee gut community, the gut microbiota dysbiosis caused by high dosage of antibiotic treatment has a cascade effect on the gut community of the nestmates in honeybee colonies.

Keywords: Apis mellifera, gut community dysbiosis, tetracycline, pass-on effect, antibiotic residue

\section{INTRODUCTION}

Honey bee (Apis spp.) is one of the most important insect pollinators, contributing an estimated 153 billion euros to the world's agriculture in 2005 (Gallai et al., 2009). The elevated loss of honey bee colonies around the world (Van der Zee et al., 2015; Brodschneider et al., 2016, 2018) poses a great threat to the food security of the world. Besides pathogens, parasites, nutritional and environmental factors, gut microbiota has been recently proved to be another important factor associated with honey bee health (Clemente et al., 2012; Brodschneider et al., 2018). In contrast to many other animals, honey bees have a relatively simple and conservative gut microbiota community which is dominated by only eight core bacterial phylotypes, including Snodgrassella alvi, Gilliamella apicola, Lactobacillus spp., Bifidobacterium spp., Frischella perrara, Bartonella apis, Parasaccharibacter 
apium and Commensalibacter spp. (Kwong et al., 2017a). These gut bacteria contribute to honey bee health in multiple ways, e.g., regulating host hormonal signaling (Zheng et al., 2017), participating in food digestion (Kesnerova et al., 2017; Zheng et al., 2019), priming the immune system against pathogenic infections (Emery et al., 2017; Kwong et al., 2017b) and promoting the endogenous detoxification (Wu et al., 2020b).

Antibiotics are used in beekeeping practice to treat or prevent bacterial infections (Piva et al., 2020), such as American foulbrood disease and European foulbrood disease, which are caused by Paenibacillus larvae and Melissococcus pluton (Arbia and Babbay, 2011), respectively. However, it has been revealed that the application of tetracycline resulted in major changes in community size structure and led to decreased survivorship of honeybees (Raymann et al., 2017, 2018). Recently, Li et al. (2017) revealed that penicillin-streptomycin treatment weakened bacterial activity in honeybees, which may negatively affect expression levels of genes encoding antimicrobial peptides and increase the honey bee's vulnerability to Nosema infection. These studies provided important insights into how antibiotic treatment affects honey bee gut community and health, while they mainly focused on the direct impacts on the individuals that received the antibiotic treatment. As a social insect, honey bee colony is rooted in a fundamental biological phenomenon known as superorganism resilience, that is the ability of a colony to sustain the loss or impairment of individuals without compromising its overall health (Sponsler and Johnson, 2017). For example, Henry et al. (2015) found that honey bee colonies exposed to neonicotinoidtreated oilseed rape suffered an increased mortality rate in the adult, but prevented any detec e change in overall colony growth or honey storage by producing more new workers. Thus, it is vital to investigate the possible passon effect of the impact of antibiotics on honey bee gut community, especially on the development of gut community of their nestmates.

The main social transmission routes of honeybee gut microbiota include fecal-oral transmission, oral-oral transmission and contact with the hive components (comb, honey, and beebread), of which fecal-oral transmission is the most important route for the establishment of a typical gut microbiota community in the receiver (Powell et al., 2014). In this study, we evaluated the possible social transmission of the effects of antibiotic treatment from two aspects. Firstly, the pass-on effects of antibiotic treatment on honeybee gut microbiota were investigated via feeding newly emerged workers with antibiotic-treated gut community. Secondly, given that the antibiotic residue after the treatment (Matsuka and Nakamura, 1990; Martel et al., 2006) may also negatively influence the colonization of normal gut microbiota, we applied a field relative level of tetracycline $(10 \mu \mathrm{g} / \mathrm{mL})$ to newly emerged workers and investigated its possible impact on the establishment of honey bee gut microbiota. In addition, we also monitored the mortality of honey bee workers to reveal any potential effect of the treatments on honey bee physiology.

\section{MATERIALS AND METHODS}

\section{Rearing of Honey Bee}

Honey bees (Apis mellifera) were kept in the experimental apiary of the Honey Bee Research Laboratory, College of Animal Sciences, Zhejiang University. Four different colonies were used as four experimental replicates (referred as E1-E4). The laboratory worker bees were raised in cages (A cylinder with a top circle diameter of $5 \mathrm{~cm}$, a bottom circle diameter of $7.5 \mathrm{~cm}$, and a height of $10 \mathrm{~cm}$ ) and incubated at $31^{\circ} \mathrm{C} \pm 1^{\circ} \mathrm{C}$ and $75 \% \pm 5 \%$ relative humidity $(\mathrm{RH})$. Dead bees were counted and removed daily. All pollen and syrup supplied to workers were irradiated and sterilized.

\section{Preparation of Honey Bee Gut Bacteria for Artificial Colonization}

Five nurse workers were sampled from a hive and placed in a sterile centrifuge tube. They were soaked in $75 \%$ ethanol for $3 \mathrm{~min}$ and then washed 3 times with sterile water. After that, their guts were immediately dissected with high-temperature sterilized tweezers under sterile conditions and homogenized together in $1 \mathrm{~mL}$ Phosphate Buffered Saline (PBS). The gut homogenate was centrifuged at $10,000 \times g$ for $10 \mathrm{~min}$ and the supernatant were removed to eliminate the possible virus contamination. Then $1 \mathrm{~mL}$ PBS was used to resuspend the bacteria and $100 \mu \mathrm{L}$ suspension was added and mixed with $2 \mathrm{mg}$ sterilized pollen.

\section{Direct Treatment of Antibiotic on Honey Bee Workers}

Antibiotic treated (AT) workers and conventional gut community $(\mathrm{CV})$ workers were obtained using a protocol described by Raymann et al. (2017). Sealed brood combs containing emerging adult workers were removed from the same hive as above and placed in an incubator at $34^{\circ} \mathrm{C} \pm 1^{\circ} \mathrm{C}$ with $80 \% \pm 5 \% \mathrm{RH}$ overnight. In the following day, newly emerged workers were randomly assigned to AT or CV groups (100 per cage). In each cage, workers were supplied with pollen containing honey bee gut microbiota for 5 days to establish a normal gut community. Then, AT workers were treated by providing ad libitum tetracycline solution at $450 \mu \mathrm{g} / \mathrm{mL}(450 \mu \mathrm{g} / \mathrm{mL}$ Tetracycline in filter-sterilized $0.5 \mathrm{M}$ sucrose solution) for another 5 days, whereas $\mathrm{CV}$ workers were only fed with $0.5 \mathrm{M}$ sucrose solution for 5 days. Afterward, bees were immobilized at $4^{\circ} \mathrm{C}$ and marked with red and green paint pens for the AT and $\mathrm{CV}$ workers, respectively, and then returned to their original colony. Two days later, $15 \mathrm{CV}$ and 15 AT workers were sampled again, of which 5 were used for $16 \mathrm{~S}$ rRNA sequencing, 5 for quantification of bacterial loads, and the remaining 5 workers were sampled to prepare normal or dysbiotic gut bacteria for downstream experiments.

\section{Evaluation of the Pass-on Effect of Honey Bee Gut Community Disturbance Induced by Antibiotic Treatment}

Germ-free workers were obtained using the protocol described by Zheng et al. (2017). Briefly, late-stage pupae, with pigmented 
eyes but lacking movement, were removed from brood frames to sterile dishes using high-temperature sterilized tweezers. The dishes were placed in an incubator at $34^{\circ} \mathrm{C} \pm 1^{\circ} \mathrm{C}$ with $80 \% \pm 5 \% \mathrm{RH}$ until bees emerged. These newly emerged germ-free bees were randomly assigned to 4 different treatment groups (30 workers per cage): (1) Workers colonized with normal gut community (referred as Nor); (2) Workers colonized with dysbiotic gut community (referred as Dys); (3) Workers colonized with normal gut community and treated with $10 \mu \mathrm{g} / \mathrm{mL}$ tetracycline (referred as NorA); (4) Workers colonized with dysbiosis gut community and treated with $10 \mu \mathrm{g} / \mathrm{mL}$ tetracycline (referred as DysA). On Day 6 post colonization, 5 workers from each group were sampled for 16S rRNA sequencing, 5 were used for quantification of bacterial loads, and rest of the workers were kept until Day 20 post colonization, to evaluate their survival rate.

\section{Gut Microbiota Analysis}

Bacterial genomic DNA was extracted using the TIANamp Stool DNA Kit (Tiangen Biotech Co., Ltd., Beijing, China) according to the manufacturer's protocol. Polymerase chain reactions (PCR) targeting the V4 regions of the 16S rDNA genes were carried out using the specific primer with the barcode. Sequencing libraries were generated using the NEBNext Ultra DNA Library Prep Kit (NEB, United States) following manufacturer's recommendations and index codes were added. The library quality was assessed on the Qubit@2.0 Fluorometer (Thermo Fisher Scientific, Waltham, MA, United States) and Agilent Bioanalyzer 2100 system (Agilent Technologies, Santa Clara, CA, United States). The library was sequenced on an Illumina NovaSeq platform and 250 bp pairedend reads were generated. The $16 \mathrm{~S}$ rRNA gene sequences were deposited in $\mathrm{NCBI}^{1}$ Sequence Read Archive with a BioProject accession number PRJNA225925.

Paired-end reads from the original DNA fragments were merged using FLASH software (Magoc and Salzberg, 2011). Sequences were analyzed using the Quantitative Insights into Microbial Ecology (QIIME) software package (Caporaso et al., 2010). We used pick_de_novo_otus.py to pick operational taxonomic units (OTUs) by making an OTU table. Sequences with $\geq 97 \%$ similarity were assigned to the same OTUs. We picked a representative sequence for each OTU and used the RDP classifier to annotate taxonomic information for each representative sequence. OTUs abundance information were normalized using a standard of sequence number corresponding to the sample with the least sequences. Subsequent analysis of alpha diversity and beta diversity were performed basing on the normalized data. Beta diversity was assessed using the Unweighted Pair-group Method followed by principal coordinate analysis (PCoA).

\section{Quantification of Bacterial Loads in the Gut of Honey Bees}

Bacterial loads of workers were determined by quantitative PCR (qPCR) using universal bacterial 16S rRNA

${ }^{1}$ https://www.ncbi.nlm.nih.gov/ primers (F: 5'-AGAGTTTGATCCTGGCTCAG-3,' R: 5' CTGCTGCCTCCCGTAGGAGT-3'). Sampled guts of workers were obtained according to the protocol described by $\mathrm{Wu}$ et al. (2020a) and their DNA using TIANamp Stool DNA Kit (Tiangen Biotech Co., Ltd., Beijing, China) according to the manufacturer's protocol. The StepOne Plus real time PCR system was used for the absolute quantification of 16S rRNA copy number. The thermal cycling conditions were as follows: the initial degeneration step was 40 amplification cycles of $95^{\circ} \mathrm{C} 30 \mathrm{~s}, 95^{\circ} \mathrm{C} 5 \mathrm{~s}$ and $60^{\circ} \mathrm{C}$ annealing $30 \mathrm{~s}$. And melt curve analysis from $60^{\circ} \mathrm{C}$ to $95^{\circ} \mathrm{C} 0.5^{\circ} \mathrm{C} / 5 \mathrm{~s}$ to determine the expected dissociation curve.

\section{Data Analysis}

SPSS software version 22.0 (IBM SPSS Statistics; Chicago, IL, United States) and GraphPad Prism 8 (GraphPad Software, San Diego, CA, United States) were used for statistical analysis. After verifying variance homogeneity and data normality, independent sample $t$-test or Mann-Whitney test was used to calculate the statistical difference of bacterial load and the alpha diversity among groups. Survival analysis was performed using KaplanMeier survival curve and log-rank test. All graphs were generated in GraphPad Prism 8.

\section{RESULTS}

\section{Direct Exposure to Tetracycline Caused Significant Disruption of Gut Community of Honey Bee}

The absolute abundance of gut bacteria was significantly reduced in antibiotic treated (AT) workers compared to conventional gut community $(\mathrm{CV})$ workers in all the four replicates (Figure 1A, student's $t$-test: for $\mathrm{E} 1: t=3.14 \mathrm{df}=8, p=0.0138$; for $\mathrm{E} 2$ : $t=2.151$, $\mathrm{df}=8, p=0.0079$; for $\mathrm{E} 3: t=2.894, \mathrm{df}=8, p=0.0201$; for $\mathrm{E} 4$ : $t=3.158, \mathrm{df}=8, p=0.0134)$. 16s rDNA amplicon sequencing demonstrated that Lactobacillus, Frischella, Commensalibacter, Bartonella, Snodgrassella and Gilliamella are the core bacterial species dominating in CV workers and AT workers (Figure 1B). The application of tetracycline did not remove any of these core bacteria completely, but caused a general decreasing trend of these bacteria (Supplementary Figure 1). For example, the absolute abundance of Snodgrassella was significantly decreased in AT workers in E1, E3 and E4, and showed a decreasing trend in E2 (Figure 1C, student's $t$-test: for E1: $t=3.159 \mathrm{df}=8, p=0.0134$; for $\mathrm{E} 2: t=1.689 \mathrm{df}=8, p=0.1298$; for E3: $t=2.669$, $\mathrm{df}=8$, $p=0.0284$; for E4: $t=2.656$, $\mathrm{df}=8, p=0.029$ ). The absolute abundance of Lactobacillus was significantly decreased in AT workers in E1 and E3, and showed a decreasing trend in E2 and E4 (Figure 1D, student's $t$-test: for $\mathrm{E} 1: t=3.464$, $\mathrm{df}=8, p=0.0085$; for $\mathrm{E} 2: t=1.920$, $\mathrm{df}=8, p=0.0912$; for $\mathrm{E} 3: t=3.977 \mathrm{df}=8$, $p=0.0041$; for E4: $t=1.809 \mathrm{df}=8, p=0.1080$ ). However, no significant difference was found on the alpha diversity (Shannon index and Chao 1 index) of microbiota species between CV workers and AT workers in any of the replicates (Figure 2A). Principal coordinate analysis (PCoA, using bray-curtis distance) 

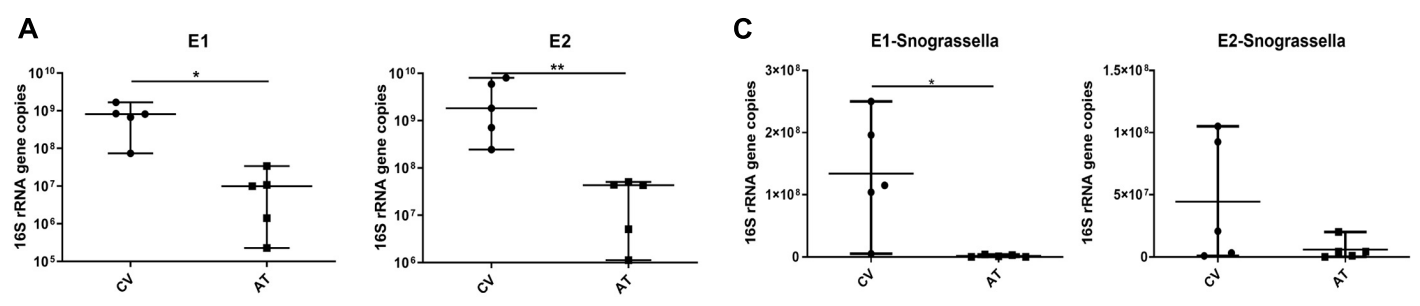

E3
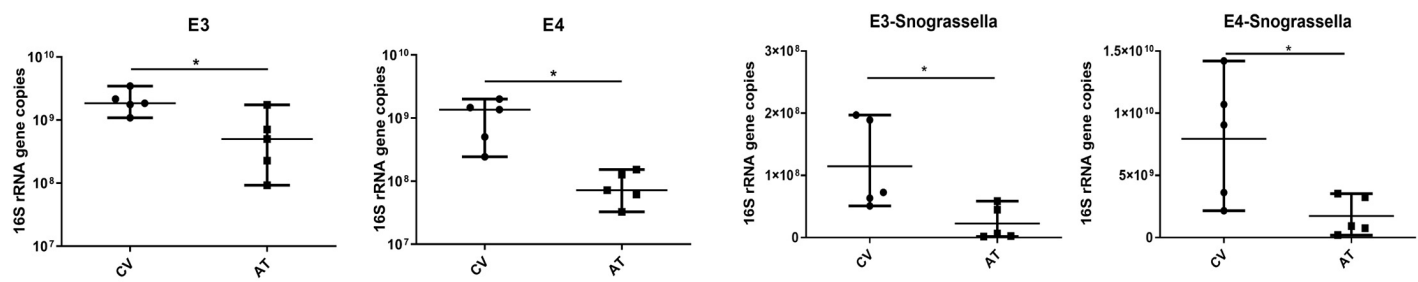

B

E1

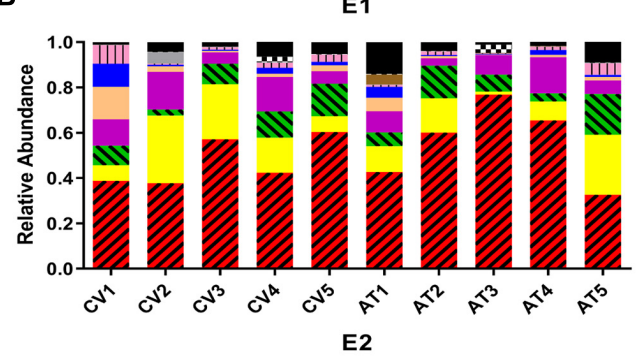

- Others

\$. Pantoea

- Melissococcus

Snodgrassella

- unidentified_Enterobacteriar

- Gilliamella

Iv Commensalibacter

- Bartonella

- Bombella

2 Lactobacillus

IIII Frischella

- Others

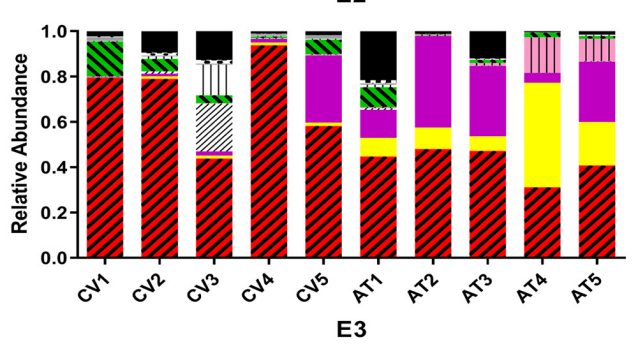

$\infty$ Bombella

x Serratia

Melissococcus

II Denitratisoma

(v Commensalibacter

III Frischella

VII/. Candidatus_Kuenenia

- Gilliamella

Snodgrassella

ca Lactobacillus

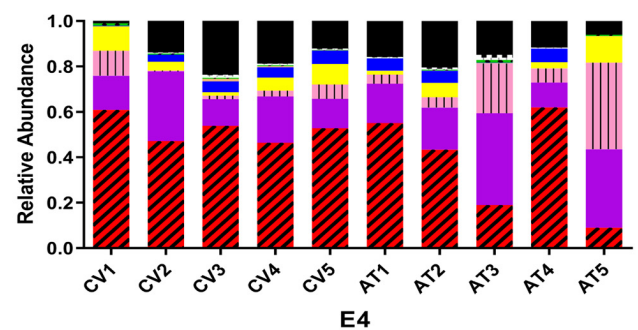

athers

Streptomyces

1) RB41

Comamonas

w Commensalibacter

Hydrogenophilus

- Bifidobacterium

- Snodgrassella

III Frischella

Gilliamella

Lactobacillus

E4

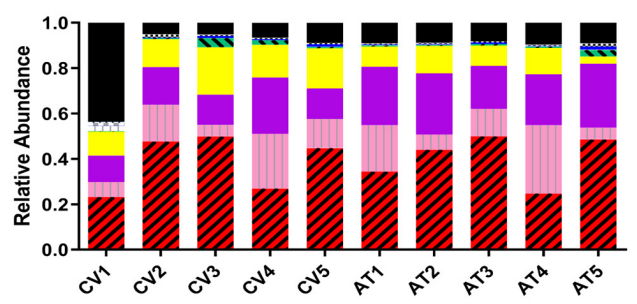

- Others

III Rubrobacter

Sphingomonas

« Bombella

- Bifidobacterium

RB41

w Commensalibacter

Snodgrassella

- Gilliamella

111II Frischella

04
D

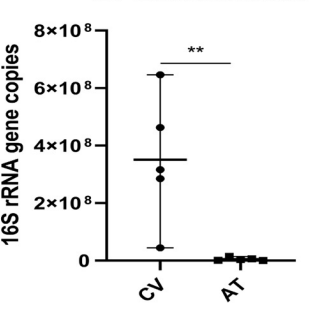

E2-Lactobacillus

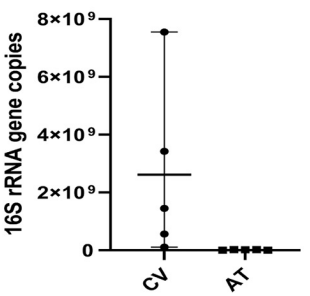

E3-Lactobacillus

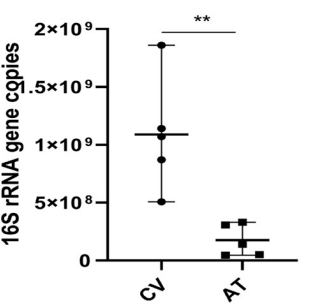

E4-Lactobacillus

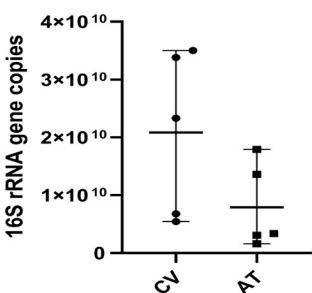

FIGURE 1 | Changes of honey bee gut microbiota after tetracycline treatment. (A) The absolute abundance of gut bacteria of CV and AT workers ( $n=5)$, total bacterial 16S rRNA gene copies were estimated by qPCR. (B) Stacked column graph showing the relative abundance of bacterial species in CV and AT workers. (C) The absolute abundance of Snodgrassella in CV and AT workers $(n=5)$. (D) The absolute abundance of Lactobacillus in CV and AT workers $(n=5)$. ${ }^{*} p<0.05$, ${ }^{* \star} p<0.01$, student's $t$-test. CV, conventional gut community workers, AT, Antibiotic treated workers. 


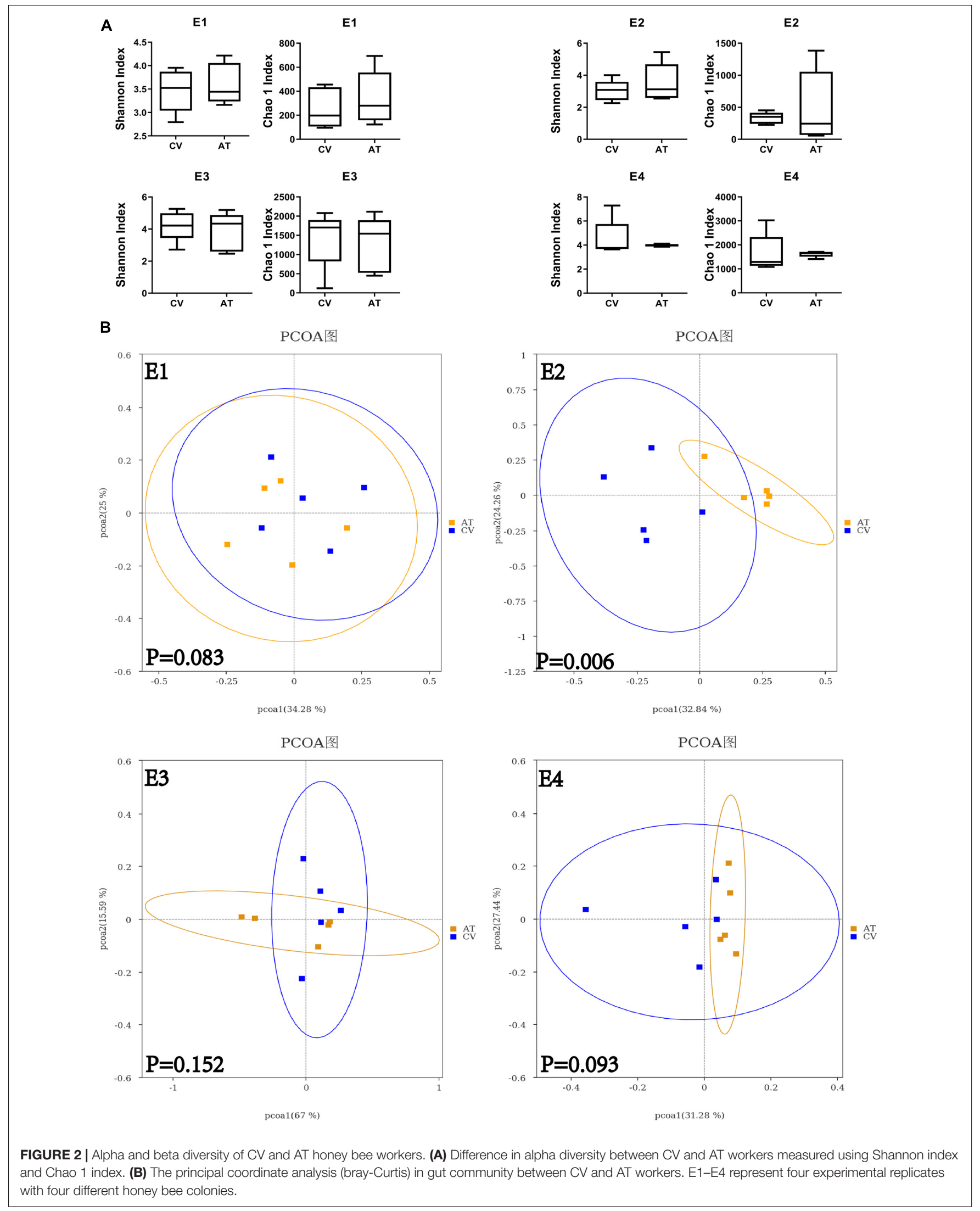


showed that gut community compositions of AT workers were dramatically changed in contrast to CV workers in E2 (Figure 2B, Anosim analysis, $p=0.006$ ), but not in other 3 replicates.

\section{Tetracycline-Induced Gut Microbiome Disequilibrium Passed to Their Receivers}

To reveal the possibility of pass-on effect to their nestmates of tetracycline-treated gut microbiota disequilibrium, we determined the gut microbiota composition and size of workers that were exposed to either normal or dysbiotic gut community. Quantification with 16s rDNA copy numbers revealed that the total bacterial abundances of these workers were not influenced by the type of gut microbiota they received (Figure 3A), but 16s rDNA amplicon sequencing analyses of the gut microbiota revealed that the compositions of their gut community were significantly influenced. The overall community structure showed that the gut microbiome of these workers in different treatment groups were dominated by six genera in our samples, namely, Lactobacillus, Bifidobacterium, Snodgrassella, Bartonella, Gilliamella, and Frischella (Figure 3B). The species diversity comparisons with Shannon index did not show significant difference between the groups of Workers colonized with normal gut community (Nor) and Workers colonized with dysbiotic gut community (Dys) (Figure 4A) in any of the four replicates, while species richness comparisons with Chao 1 index showed significant difference between the two groups in two replicates (E3 and E4) (student's $t$-test: for E3: $t=3.464, \mathrm{df}=8, p=0.0085$; for E2: $t=1.920, \mathrm{df}=8$, $p=0.0912)$. There were no significant differences in Shannon index and Chao 1 index between the NorA and the DysA in the four experiments. PCoA analysis revealed that the overall microbiota structure was significantly different according to the type of gut bacteria they received, as samples of Nor and Dys formed two distinctive cluster in three of the four replicates (Figure 4B, Anosim analysis: for E1: $p 1=0.021$, for $\mathrm{E} 2: p 1=0.004$, for $\mathrm{E} 3: p 1=0.118$, for $\mathrm{E} 4: p 1=0.009)$ and samples of NorA and DysA formed two distinctive cluster in two of the four replicates (Figure 4B, Anosim analysis: for E1: $p=0.058$, for E2: $p=0.028$, for E3: $p=0.027$, for $\mathrm{E} 4$ : $p=0.302$ ).

To evaluate the possible negative impact of low dosage antibiotic residue on the establishment of honey bee gut microbiota, $10 \mu \mathrm{g} / \mathrm{mL}$ tetracycline was used to treat workers during the development of their gut communities. However, no significant impact was observed, as neither the community size nor the makeup was significantly different between Nor and NorA, or between Dys and DysA (Figures 3A, 4A).

In addition, we evaluated and compared the longevity of workers from different group, and the survival rates of bees of Dys, NorA or DysA were not significantly different from that of control group (Supplementary Figure 2).

The linear discriminant analysis effect size (LEfSe) algorithm was then applied to determine which microbiota taxa caused the significant difference between Nor workers and Dys workers. Significant differences on the relative abundances of some taxa were found, but the taxa showing differences were not consistent in the four replicates (Figures 5A-D). For example, in E1, Dys workers showed higher phylum_Proteobacteria relative abundance,but lower phylum_Firmicutes and genus_Gilliamella relative abundance. In E2, Dys workers harbored lower genus_Snodgrassella and genus_Gilliamella relative abundance. In E3, Dys workers harbored higher class_Gammaproteobacteria and phylum_Proteobacteria relative abundance. And in E4, Dys workers harbored higher phylum_Firmicutes, genus_Lactobacillus and genus_Frischella relative abundance but lower phylum_Proteobacteria, genus_Snodgrassella and genus_Bombella.

\section{DISCUSSION}

Antibiotics have been widely used in animals for disease control and have significantly contributed to the development of animal husbandry industries. However, the collateral damage caused by antibiotic treatment has also been a great concern (Holzheimer, 2001; Martel et al., 2006; Modi et al., 2014; Becattini et al., 2016). The application of antibiotics in honey bees may not only cause damage to the individuals that received the application but also to their nestmates via certain pathways due to the sociality of honey bees. Therefore, we investigated the effect of honeybee gut microbiota dysbiosis caused by tetracycline treatment on the establishment of gut community of their nestmates.

Tetracycline treatment had persistent effects on both the size and composition of A. mellifera gut microbiota and reduced genetic diversity of core species in A. mellifera gut community (Raymann et al., 2017, 2018). In our study, tetracycline treatment also significantly reduced the absolute abundance of gut bacteria in honey bee workers. At the same time, tetracycline treatment significantly changed the overall microbiota structure in E2, and the same trend was observed in the other 3 replicates. These results suggest that both the $\mathrm{CV}$ and AT worker models were successfully established for the downstream experiments. However, unlike previous findings (Li et al., 2017; Raymann et al., 2017), the application of tetracycline did not cause significant difference in microbiota diversity within individual hosts (alpha diversity) in our study. This may be explained by the difference of incidence of antibiotic resistance, as the usage of antibiotics could be different between regions and apiaries.

The major contributors of honey bee microbiota are transmitted via direct contact during the first social interactions with hive mates (Engel and Moran, 2013; Powell et al., 2014). The gut microbiota of tetracycline-treated honey bee workers did not recover after being reintroduced to their colonies (Raymann et al., 2017), indicating a high possibility of cascade effect to their nestmates of the tetracycline treatment. We investigated this cascade effect in lab condition via artificially inoculating tetracycline-treated gut microbiota to newly emerged workers. Despite non-significant effect on the absolute amount and alpha diversity of receiver's gut microbiota, beta diversity was found to be significantly altered in Dys workers compared to the Nor workers inoculated with normal gut microbiota in 3 of the 4 replicates. This result 


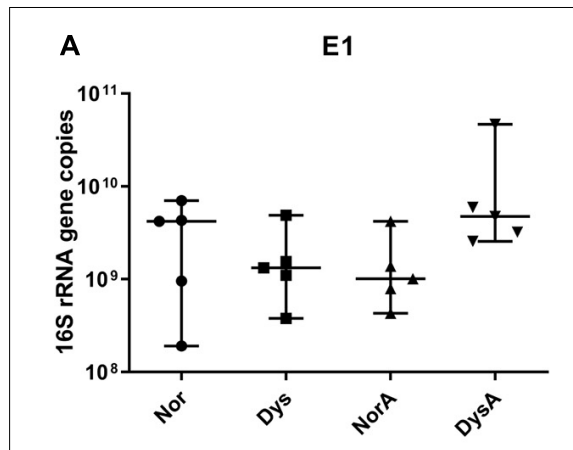

E2

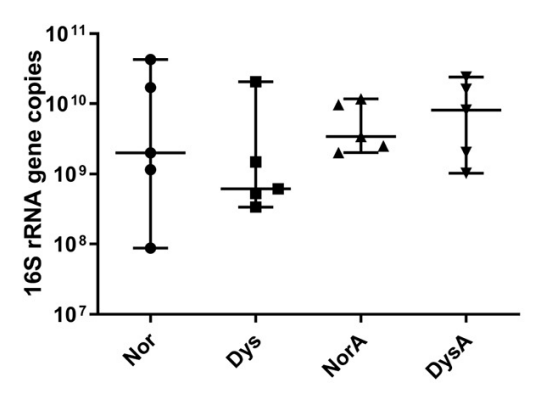

E3

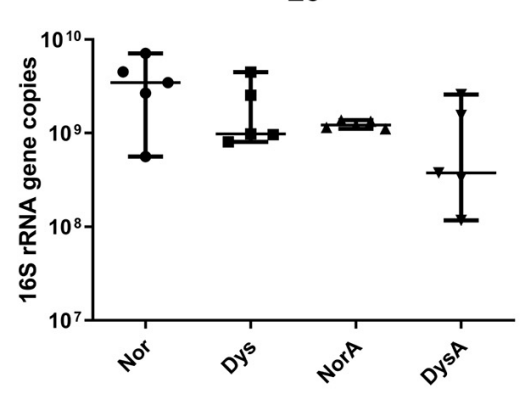

E4

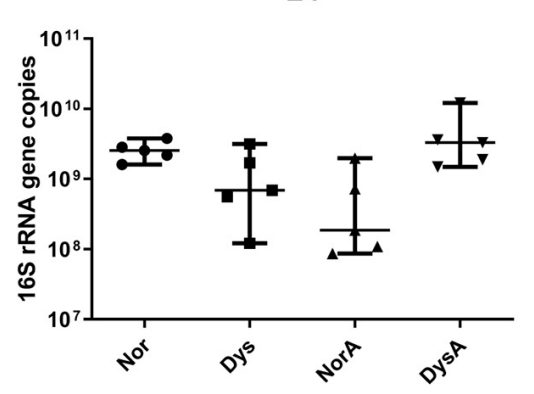

B

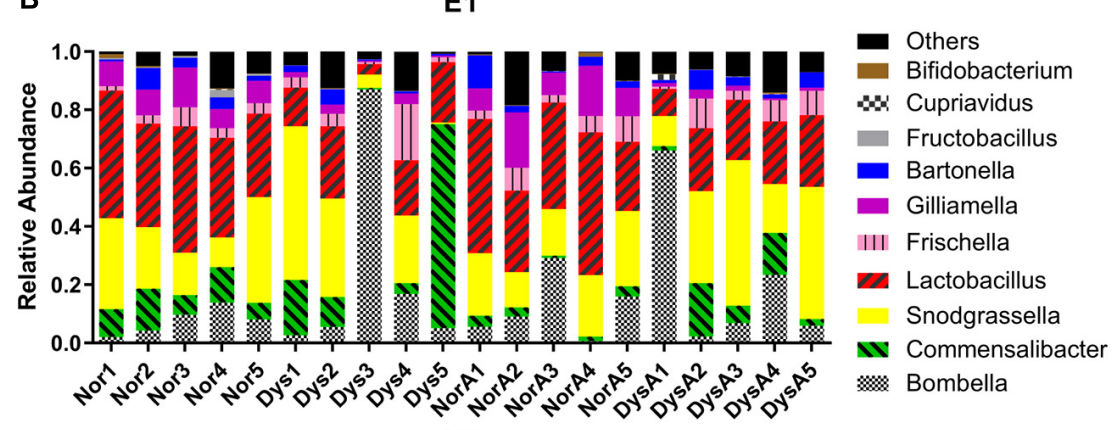

E2

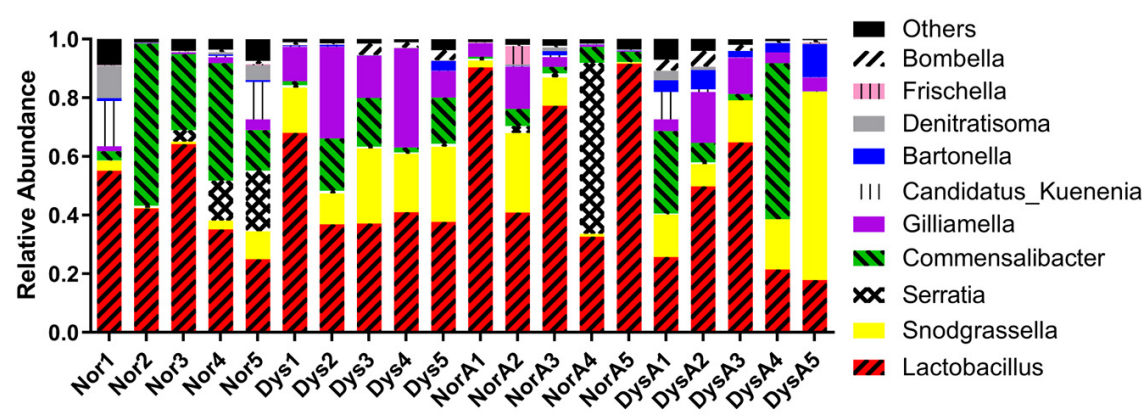

E3

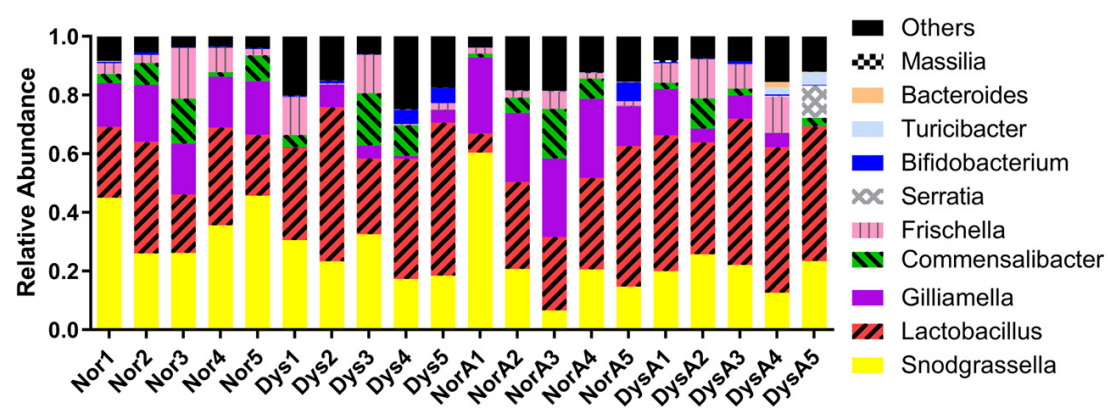

E4

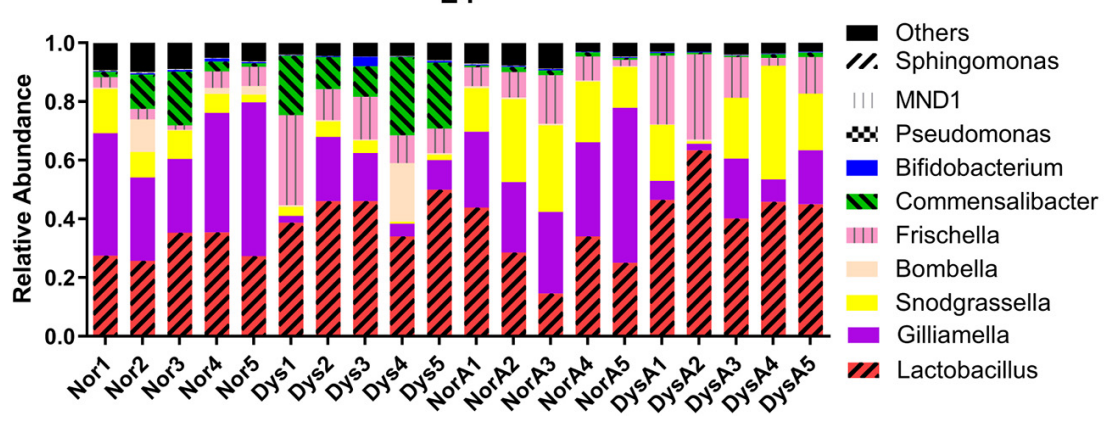

FIGURE 3 | Size and Composition of gut bacteria of Nor, Dys, NorA, and DysA honey bee workers. (A) The absolute abundance of gut bacteria of Nor, Dys, NorA and DysA workers $(n=5)$, total bacterial 16S rRNA gene copies were estimated by qPCR. (B) Stacked column graph showing the relative abundance of bacterial species in Nor, Dys, NorA, and DysA workers. Nor, workers colonized with normal gut community, Dys, workers colonized with dysbiotic gut community, NorA, workers colonized with normal gut community and treated with $10 \mu \mathrm{g} / \mathrm{mL}$ tetracycline, DysA, workers colonized with dysbiotic gut community and treated with $10 \mu \mathrm{g} / \mathrm{mL}$ tetracycline.

suggests that the impact of tetracycline treatment on gut microbiota may be transmitted to other individuals. Various functions have been found for each of the core species of honey bee gut bacteria (Raymann et al., 2017) and functional redundancy among them stabilize the general function of gut microbiota in honey bees ( $\mathrm{Wu}$ et al., 2021). Therefore, the disruption of gut microbiota indirectly caused by tetracycline treatment may negatively affect the function of gut microbiota 


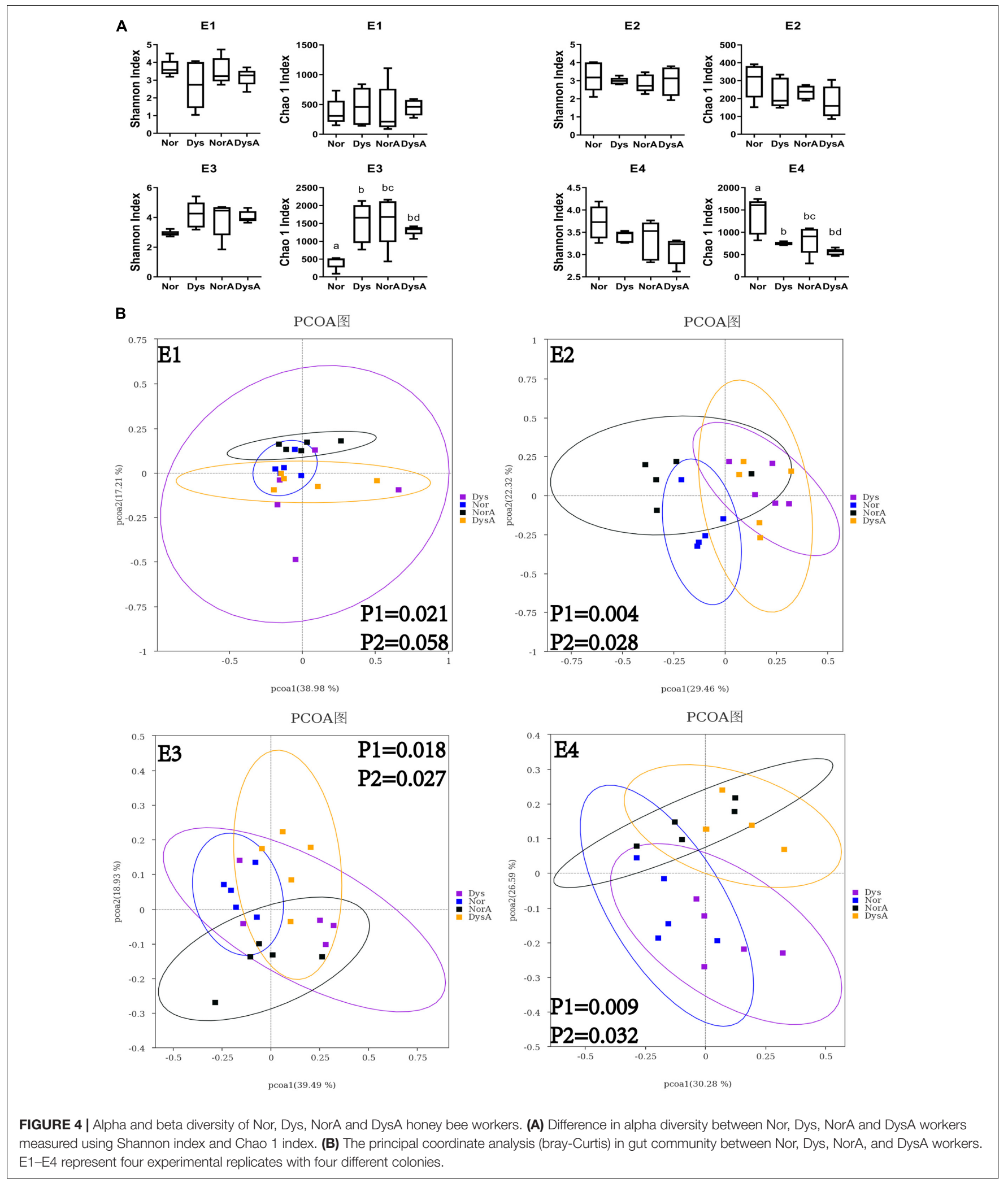

and subsequently the physiology of their host, despite that no difference was observed on the mortality of different groups in our study.
LEfSe analysis identified variable microbial taxa that differentiated the groups in each replicate, suggesting variations of this cascade effect among cases. The presence 


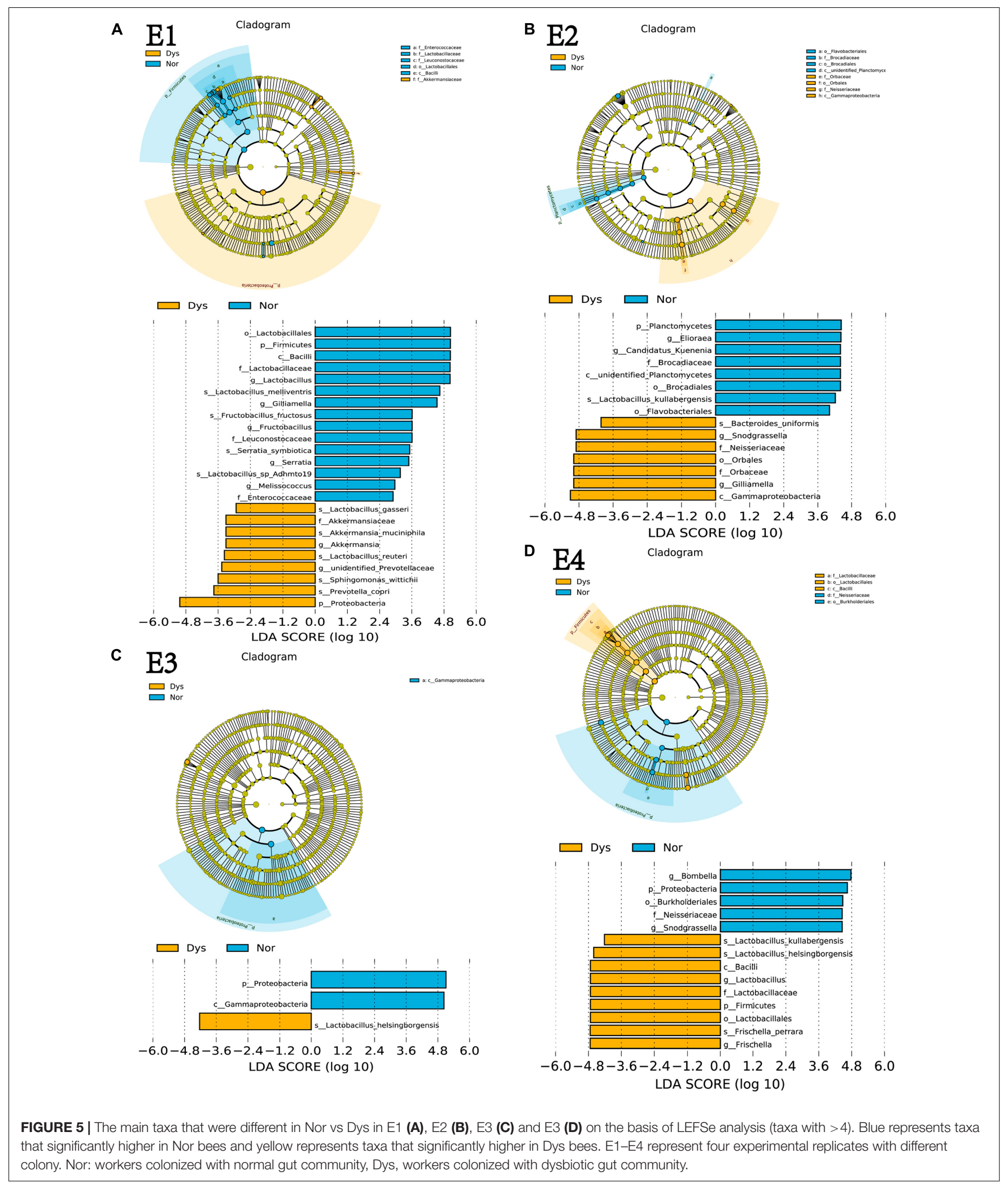

of the core species is quite consistent in honey bee gut microbiota, while their relative abundances vary at individual and colony levels in honeybees (Powell et al., 2014;
Dong et al., 2020). It is therefore not surprising that the results vary among replicates. This also suggests that multiple sources of gut microbiota and honey 
bees are necessary in this kind of research on honey bee gut microbiota.

The antibiotic residue in bee hive is inevitable after application. A previous study has shown that the tetracycline hydrochloride could be detected in honey 146 days after treatment in a colony (Martel et al., 2006). Thus, the possible negative impact of low concentration antibiotic on the development of honey bee gut microbiota and survival rate of bees is worthy of attention. No significant effect was found in this study possibly due to the low dosage treatment and/or the antibiotic resistance of bacteria. Interestingly, when the low dosage treatment was applied simultaneously, beta diversity was also found to be significantly altered in 2 of the 4 replicates when bees received tetracycline-treated gut microbiota. This further confirmed the pass-on effect of high dosage tetracycline treatment.

\section{CONCLUSION}

Our findings clearly demonstrated that direct exposure to tetracycline caused significant disruption of gut community of honey bee workers and the disorder has a cascade effect on the workers inoculated with the antibiotic-treated gut microbiota. This pass-on effect of antibiotic treatment represents a new negative impact of antibiotics in honey bee colonies and further study is needed to monitor the constant exposure to antibiotic along with its potential damage to honey bee health.

\section{DATA AVAILABILITY STATEMENT}

The datasets presented in this study can be found in online repositories. The names of the repository/repositories

\section{REFERENCES}

Arbia, A., and Babbay, B. (2011). Management strategies of honey bee diseases . J. Entomol. 8, 1-15. doi: 10.3923/je.2011.1.15

Becattini, S., Taur, Y., and Pamer, E. G. (2016). Antibiotic-induced changes in the intestinal microbiota and disease. Trends Mol. Med. 22, 458-478. doi: 10.1016/ j.molmed.2016.04.003

Brodschneider, R., Gray, A., Adjlane, N., Ballis, A., Brusbardis, V., Charrière, J.D., et al. (2018). Multi-country loss rates of honey bee colonies during winter 2016/2017 from the COLOSS survey. J. Apicult. Res. 57, 452-457. doi: 10.1080/ 00218839.2018.1460911

Brodschneider, R., Gray, A., van der Zee, R., Adjlane, N., Brusbardis, V., Charrière, J.-D., et al. (2016). Preliminary analysis of loss rates of honey bee colonies during winter 2015/16 from the COLOSS survey. J. Apicult. Res. 55, 375-378. doi: 10.1080/00218839.2016.1260240

Caporaso, J. G., Kuczynski, J., Stombaugh, J., Bittinger, K., Bushman, F. D., Costello, E. K., et al. (2010). QIIME allows analysis of high-throughput community sequencing data. Nat. Methods 7, 335-336. doi: 10.1038/nmeth.f. 303

Clemente, J. C., Ursell, L. K., Parfrey, L. W., and Knight, R. (2012). The impact of the gut microbiota on human health: an integrative view. Cell 148, 1258-1270. doi: 10.1016/j.cell.2012.01.035

Dong, Z. X., Li, H. Y., Chen, Y. F., Wang, F., Deng, X. Y., Lin, L. B., et al. (2020). Colonization of the gut microbiota of honey bee (Apis mellifera) workers at different developmental stages. Microbiol. Res. 231:126370. doi: 10.1016/j. micres.2019.126370 and accession number(s) can be found in the article/ Supplementary Material.

\section{AUTHOR CONTRIBUTIONS}

SJ, YW, FH, and HZ conceived and designed the study. SJ, YW, GC, and SW performed experiments. SJ and YW analyzed data and drafted the manuscript. All authors edited and approved the final version of the manuscript.

\section{FUNDING}

This study was funded by the National Natural Science Foundation of China (32072798, HZ and 31902222, YW), Science and Technology Department of Zhejiang Province, China (2016C02054-11, FH), and China Agriculture Research System of MOF and MARA (CARS44, FH and HZ).

\section{ACKNOWLEDGMENTS}

We thank the Experimental Teaching Centre, College of Animal Sciences, Zhejiang University for providing access to their instruments.

\section{SUPPLEMENTARY MATERIAL}

The Supplementary Material for this article can be found online at: https://www.frontiersin.org/articles/10.3389/fmicb. 2021.781746/full\#supplementary-material

Emery, O., Schmidt, K., and Engel, P. (2017). Immune system stimulation by the gut symbiont Frischella perrara in the honey bee (Apis mellifera). Mol. Ecol. 26, 2576-2590. doi: 10.1111/mec. 14058

Engel, P., and Moran, N. A. (2013). The gut microbiota of insects - diversity in structure and function. FEMS Microbiol. Rev. 37, 699-735. doi: 10.1111/15746976.12025

Gallai, N., Salles, J.-M., Settele, J., and Vaissière, B. E. (2009). Economic valuation of the vulnerability of world agriculture confronted with pollinator decline. Ecol. Econ. 68, 810-821. doi: 10.1016/j.ecolecon.2008.06.014

Henry, M., Cerrutti, N., Aupinel, P., Decourtye, A., Gayrard, M., Odoux, J. F., et al. (2015). Reconciling laboratory and field assessments of neonicotinoid toxicity to honeybees. Proc. Biol. Sci. 282:20152110. doi: 10.1098/rspb.2015.2110

Holzheimer, R. G. (2001). Antibiotic induced endotoxin release and clinical sepsis: a review. J. Chemother. 13 Spec No.1, 159-172. doi: 10.1179/joc.2001.13. Supplement-2.159

Kesnerova, L., Mars, R. A. T., Ellegaard, K. M., Troilo, M., Sauer, U., and Engel, P. (2017). Disentangling metabolic functions of bacteria in the honey bee gut. PLoS Biol. 15:e2003467. doi: 10.1371/journal.pbio.2003467

Kwong, W. K., Mancenido, L. A., Koch, H., Sing, K.-W., Soh, E. J. Y., Ascher, J. S., et al. (2017a). Dynamic microbiome evolution in social bees. Sci. Adv. 3:e1600513. doi: 10.1126/sciadv.1600513

Kwong, W. K., Mancenido, A. L., Moran, and N. A. (2017b). Immune system stimulation by the native gut microbiota of honey bees. R. Soc. Open Sci. 4:170003. doi: $10.1098 /$ rsos. 170003

Li, J. H., Evans, J. D., Li, W. F., Zhao, Y. Z., DeGrandi-Hoffman, G., Huang, S. K., et al. (2017). New evidence showing that the destruction of gut bacteria by 
antibiotic treatment could increase the honey bee's vulnerability to Nosema infection. PLoS One 12:e0187505. doi: 10.1371/journal.pone.0187505

Magoc, T., and Salzberg, S. L. (2011). FLASH: fast length adjustment of short reads to improve genome assemblies. Bioinformatics 27, 2957-2963. doi: 10.1093/ bioinformatics/btr507

Martel, A. C., Zeggane, S., Drajnudel, P., Faucon, J. P., and Aubert, M. (2006). Tetracycline residues in honey after hive treatment. Food Addit. Contam. 23, 265-273. doi: 10.1080/02652030500469048

Matsuka, M., and Nakamura, J. (1990). Oxytetracycline residues in honey and royal jelly. J. Apicult. Res. 29, 112-117.

Modi, S. R., Collins, J. J., and Relman, D. A. (2014). Antibiotics and the gut microbiota. J. Clin. Invest. 124, 4212-4218. doi: 10.1172/JCI72333

Piva, S., Giacometti, F., Marti, E., Massella, E., Cabbri, R., Galuppi, R., et al. (2020). Could honey bees signal the spread of antimicrobial resistance in the environment? Lett. Appl. Microbiol. 70, 349-355. doi: 10.1111/lam. 13288

Powell, J. E., Martinson, V. G., Urban-Mead, K., and Moran, N. A. (2014). Routes of acquisition of the gut microbiota of the honey bee Apis mellifera. Appl. Environ. Microbiol. 80, 7378-7387. doi: 10.1128/AEM.01861-14

Raymann, K., Bobay, L. M., and Moran, N. A. (2018). Antibiotics reduce genetic diversity of core species in the honeybee gut microbiome. Mol. Ecol. 27, 2057-2066. doi: 10.1111/mec.14434

Raymann, K., Shaffer, Z., and Moran, N. A. (2017). Antibiotic exposure perturbs the gut microbiota and elevates mortality in honeybees. PLoS Biol. 15:e2001861. doi: 10.1371/journal.pbio.2001861

Sponsler, D. B., and Johnson, R. M. (2017). Poisoning a society: a superorganism perspective on honey bee toxicology. Bee World 94, 30-32. doi: 10.1080/ 0005772x.2017.1295762

Van der Zee, R., Brodschneider, R., Brusbardis, V., Charrière, J.-D., Chlebo, R., Coffey, M. F., et al. (2015). Results of international standardised beekeeper surveys of colony losses for winter 2012-2013: analysis of winter loss rates and mixed effects modelling of risk factors for winter loss. J. Apicult. Res. 53, 19-34. doi: 10.3896/ibra.1.53.1.02

Wu, Y., Zheng, Y., Chen, Y., Chen, G., Zheng, H., and Hu, F. (2020a). Apis cerana gut microbiota contribute to host health though stimulating host immune system and strengthening host resistance to Nosema ceranae. R. Soc. Open Sci. 7:192100. doi: 10.1098/rsos.192100

Wu, Y., Zheng, Y., Chen, Y., Wang, S., Chen, Y., Hu, F., et al. (2020b). Honey bee (Apis mellifera) gut microbiota promotes host endogenous detoxification capability via regulation of $\mathrm{P} 450$ gene expression in the digestive tract. Microb. Biotechnol. 13, 1201-1212. doi: 10.1111/1751-7915.13579

Wu, Y., Zheng, Y., Wang, S., Chen, Y., Tao, J., Chen, Y., et al. (2021). Genetic divergence and functional convergence of gut bacteria between the Eastern honey bee Apis cerana and the Western honey bee Apis mellifera. J. Adv. Res. doi: 10.1016/j.jare.2021.08.002

Zheng, H., Perreau, J., Powell, J. E., Han, B., Zhang, Z., Kwong, W. K., et al. (2019). Division of labor in honey bee gut microbiota for plant polysaccharide digestion. Proc. Natl. Acad. Sci. U.S.A. 116, 25909-25916. doi: 10.1073/pnas. 1916224116

Zheng, H., Powell, J. E., Steele, M. I., Dietrich, C., and Moran, N. A. (2017). Honeybee gut microbiota promotes host weight gain via bacterial metabolism and hormonal signaling. Proc. Natl. Acad. Sci. U.S.A. 114, 4775-4780. doi: 10.1073/pnas.1701819114

Conflict of Interest: The authors declare that the research was conducted in the absence of any commercial or financial relationships that could be construed as a potential conflict of interest.

Publisher's Note: All claims expressed in this article are solely those of the authors and do not necessarily represent those of their affiliated organizations, or those of the publisher, the editors and the reviewers. Any product that may be evaluated in this article, or claim that may be made by its manufacturer, is not guaranteed or endorsed by the publisher.

Copyright (c) $2022 \mathrm{Jia}, \mathrm{Wu}$, Chen, Wang, Hu and Zheng. This is an open-access article distributed under the terms of the Creative Commons Attribution License $(C C B Y)$. The use, distribution or reproduction in other forums is permitted, provided the original author(s) and the copyright owner(s) are credited and that the original publication in this journal is cited, in accordance with accepted academic practice. No use, distribution or reproduction is permitted which does not comply with these terms. 